\title{
Elementary Trigonometric Sums related to Quadratic Residues
}

A. Laradji, M. Mignotte and N. Tzanakis

\begin{abstract}
Abdallah Laradji is a professor in the Department of Mathematics \& Statistics at King Fahd University of Petroleum \& Minerals. His main research interests are in Algebra and Number Theory.

Maurice Mignotte is a professor in the Université de Strasbourg since 1974. His main domains of interest are theory of numbers and computer algebra. In computer algebra, his focus is on inequalities and algorithms on polynomials, in number theory, he is working on Diophantine approximation and Diophantine equations.

Nikos Tzanakis is a professor at the Department of Mathematics of the University of Crete. His research is around various methods for the explicit solution of Diophantine equations, mainly in integers.
\end{abstract}

Let $p$ be an odd prime. We will study the following sums

$$
T(p)=\sqrt{p} \sum_{n=1}^{(p-1) / 2} \tan \frac{\pi n^{2}}{p}
$$

and

$$
C(p)=\sqrt{p} \sum_{n=1}^{(p-1) / 2} \cot \frac{\pi n^{2}}{p} .
$$

Die Autoren dieses Beitrags ordnen den Primzahlen $p \equiv 3(\bmod 4)$ zwei trigonometrische Summen zu, die verschiedene überraschende Eigenschaften an den Tag legen. Unter anderem kann die Klassenzahl $h(-p)$ des Zahlkörpers $\mathbb{Q}(\sqrt{-p})$ mit Hilfe der Differenz der beiden Summen ausgedrückt werden. Es zeigen sich Zusammenhänge mit quadratischen Residuen mod $p$ oder dem Legendre-Symbol mod $p$. Die Summen stehen in Beziehung zur Klassenzahlformel von Dirichlet, die verschiedene Kenngrössen von algebraischen Zahlkörpern mit dem Residuum der Dedekindschen Zetafunktion in deren Pol verbindet. Dem Leser eröffnet sich ein Bouquet von klassischen Resultaten in neuem Kleid. 
Surprisingly, we came across these sums as we were working on a certain diophantine equation. Being non specialists in the relevant area, we were impressed by the nice properties that these sums have and their elegant consequences. It is this feeling of elegance that we would like to share with our readers. As pointed out to us by Juan Carlos Peral Alonso, to whom we are grateful, these sums are closely related to the class-number formula due to Dirichlet (see (20)), sometimes called "Lebesgue's formula" -see [9], p. 179-, which "explains", in a sense, their nice properties. For those readers who are not already acquainted with the notion of class-number, a brief remark has its place. Let $D$ be a negative integer which is a fundamental discriminant, i.e. either $D \equiv 1(\bmod 4)$ and $D$ is squarefree, or $D \equiv 0(\bmod 4)$ and $D / 4$ is squarefree $\equiv 2,3(\bmod 4)$. In particular, if $p$ is a prime $\equiv 3 \bmod 4$ (we will deal with such primes in this paper), $-p$ is a fundamental discriminant. The class-number $h(D)$ has a double interpretation, as the number of reduced binary quadratic forms of discriminant $D$, and as the number of classes of fractional ideals of the quadratic number field $\mathbb{Q}(\sqrt{D})$. The reader may very well profit by reading, for example, sections 4.9.1, 5.1, 5.2 and 5.3.1 of H. Cohen's book [7], written in a very concrete way; see, especially, the conclusion following Lemma 5.3.4 therein.

All the results presented in this paper, possibly with the exception of Properties 1, 3 and $5(19)$, are scattered in the literature, mainly (but not exclusively) in articles about the classnumber of binary quadratic forms; see, for example, [9] and [16]. Therefore, our purpose is not to present new results; rather having expository-pedagogic aim, our paper offers a bouquet of classical results which are presented in a very smooth, as we believe, manner, practically using only Elementary Mathematics, or appealing to short and easily readable elementary papers, like [2], [4], [6], [18], [19].

Since $T(p)$ and $C(p)$ are very closely related to each other (see (13) and (16)), we will mainly focus on $T(p)$. We also note that our $T(p)$ is equal to H.L. Montgomery's $-T(1, \chi)$ as defined in [17], where $\chi$ is the non-trivial quadratic character.

As we will see immediately below, if $p \equiv 1(\bmod 4)$, then $T(p)=0=C(p)$, therefore, concerning the sums $T(p)$ and $C(p)$, only the case $p \equiv 3(\bmod 4)$ is of interest. For this case, we prove a number of elegant number-theoretical properties of $T(p)$. Some of them have the flavour of the well-known property of the primes $p \equiv 3(\bmod 4)$, asserting that, in the range 1 to $(p-1) / 2$ there are more quadratic residues $\bmod p$ than non-quadratic residues (see, for example, [6], [18], [19]). Further, Properties 1 and 2 below give a simple rule comparing the numbers of even and odd quadratic residues in $\{1,2, \ldots, p-1\}$ and Property 5 gives an extremely simple rule for expressing $h(-p)$, the class-number of the quadratic field $\mathbb{Q}(\sqrt{-p})$.

First, a few remarks have their place. If $Q$ is any complete set of quadratic residues $\bmod p$, we can write

$$
T(p)=\sqrt{p} \sum_{j \in Q} \tan \frac{\pi j}{p} .
$$

If $p \equiv 1(\bmod 4)$, then $-Q \equiv Q(\bmod p)$, from which we immediately conclude that $T(p)=0$ and, similarly, $C(p)=0$. Therefore we make the following assumption:

Throughout this paper, $p$ will always denote a prime $\equiv 3(\bmod 4)$ 
We denote by $\zeta$ a primitive $p$-root of unity and we put $i=\sqrt{-1}$. Also, by $\sqrt{p}$ we mean the positive square root of $p$. It is easy to see that

$$
T(p)=i \sqrt{p} \sum_{j \in Q} \frac{1-\zeta^{j}}{1+\zeta^{j}}
$$

therefore, we have

$$
\begin{aligned}
T(p) & =i \sqrt{p} \sum_{j=1}^{(p-1) / 2} \frac{1-\zeta^{j^{2}}}{1+\zeta^{j^{2}}}=i \sqrt{p} \sum_{j=1}^{(p-1) / 2}\left(\frac{2}{1+\zeta^{j^{2}}}-1\right) \\
& =i \sqrt{p} \sum_{j=1}^{(p-1) / 2}\left(\frac{1+\left(\zeta^{j^{2}}\right)^{p}}{1+\zeta^{j^{2}}}-1\right) \\
& =i \sqrt{p} \sum_{j=1}^{(p-1) / 2}\left(1-\zeta^{j^{2}}+\left(\zeta^{j^{2}}\right)^{2}-\cdots+\left(\zeta^{j^{2}}\right)^{p-1}-1\right) \\
& =i \sqrt{p} \sum_{j=1}^{(p-1) / 2}\left(-\zeta^{j^{2}}+\left(\zeta^{j^{2}}\right)^{2}-\cdots+\left(\zeta^{j^{2}}\right)^{p-1}\right) \\
& =\frac{i \sqrt{p}}{2} \sum_{j=1}^{p-1}\left(-\zeta^{j^{2}}+\left(\zeta^{j^{2}}\right)^{2}-\cdots+\left(\zeta^{j^{2}}\right)^{p-1}\right) \\
& =\frac{i \sqrt{p}}{2} \sum_{k=1}^{p-1}(-1)^{k} \sum_{j=1}^{p-1} \zeta^{j^{2} k}=\frac{i \sqrt{p}}{2} \sum_{k=1}^{p-1}(-1)^{k} \sum_{j=0}^{p-1} \zeta^{j^{2} k}
\end{aligned}
$$

For every $k=1, \ldots, p-1, \sum_{j=0}^{p-1} \zeta^{j^{2} k}$ is the well-known Gaussian sum, denoted by $S(k, p)$, which is equal to $i\left(\frac{k}{p}\right) \sqrt{p}$, where $(\dot{p})$ is the Legendre symbol. This is a straightforward consequence of the following more general well-known result:

Let $m$ be an odd positive number and let $n$ be an integer relatively prime to $m$.

Put $S(k, m)=\sum_{j=0}^{m-1} e^{2 \pi i j^{2} k / m}$. Then,

$$
S(k, m)=\left\{\begin{array}{lll}
\left(\frac{k}{m}\right) \sqrt{m} & \text { if } m \equiv 1 \quad(\bmod 4) \\
i\left(\frac{k}{m}\right) \sqrt{m} & \text { if } m \equiv 3 & (\bmod 4)
\end{array}\right.
$$

See, e.g. Theorem 5.6 in Chapter 7 of [13]. When $m$ is a prime $p \equiv 3(\bmod 4)$, we can more directly prove that

$$
S(k, p)=i\left(\frac{k}{p}\right) \sqrt{p},
$$


without appealing to the above result, by turning to a short paper of Bamba and Chowla [2]. In that paper, an interesting brief and elementary proof of the relation $(1-i)(1+$ $\left.i^{m}\right) S(1, m)=2 \sqrt{m}$, where $m$ is a positive odd integer, is given. Consequently, if $p$ is a prime $\equiv 3(\bmod 4)$, then $S(1, p)=i \sqrt{p}$. By the definition of $S(k, m)$ it is clear that, if $k$ is a quadratic residue $\bmod p$, then $S(k, p)=S(1, p)$; and if $k$ is a quadratic non-residue, then

$$
S(k, p)=S(-1, p)=\overline{S(1, p)}=\overline{i \sqrt{p}}=-i \sqrt{p},
$$

as claimed.

Now, going back to (4) and using (5), we obtain the following expression for $T(p)$ :

$$
T(p)=\frac{p}{2} \sum_{k=1}^{p-1}(-1)^{k+1}\left(\frac{k}{p}\right) .
$$

Let $a_{1}, a_{2}, \ldots, a_{\mu}$ and $b_{1}, b_{2}, \ldots, b_{v}$ be, respectively, the even and odd quadratic residues $\bmod p$ in the set $P=\{1,2, \ldots, p-1\}$. Clearly, $\mu+v=(p-1) / 2$ and the set of the quadratic non-residues $\bmod p$ in $P$ is $\left\{p-a_{1}, \ldots, p-a_{\mu}, p-b_{1}, \ldots, p-b_{\nu}\right\}$. Note that a summand $(-1)^{k+1}\left(\frac{k}{p}\right)$ in the right-hand side of (6) is positive iff $k \in$ $\left\{p-b_{1}, \ldots, p-b_{\nu}, b_{1}, \ldots, b_{\nu}\right\}$, i.e. $2 v$ summands are positive and, analogously, $2 \mu$ summands are negative. Then, $T(p)=p(v-\mu)$, where we observe that $v-\mu$ is an odd number, since $v+\mu=(p-1) / 2$. Thus, we have the following:

Property 1. Let $p$ be a prime $\equiv 3(\bmod 4)$ and let $q_{o}(p)$ and $q_{e}(p)$ be, respectively, the number of odd and even quadratic residues $\bmod p$ in the set $\{1,2, \ldots, p-1\}$. Then

$$
T(p)=p\left(q_{o}(p)-q_{e}(p)\right)
$$

In particular, $T(p)$ is an odd integer divisible by $p$ and by no higher power of $p$.

Next, we rewrite the definition (1) of $T(p)$ as follows,

$$
T(p)=\frac{\sqrt{p}}{2} \sum_{n=1}^{p-1} \tan \frac{n^{2} \pi}{p} .
$$

We have the following inequality of A.L. Whiteman (Theorem 2 of [19]):

$$
\sum_{n=1}^{p-1} \cot \frac{n^{2} \pi}{p}>0
$$

In view of the identity $\tan \theta=\cot \theta-2 \cot 2 \theta$, the relation (8) becomes

$$
\frac{2}{\sqrt{p}} T(p)=\sum_{n=1}^{p-1} \cot \frac{n^{2} \pi}{p}-2 \sum_{n=1}^{p-1} \cot \frac{2 n^{2} \pi}{p} .
$$


Let $n=1,2, \ldots, p-1$. If $p \equiv 7(\bmod 8)$, the sets $\left\{2 n^{2}\right\}$ and $\left\{n^{2}\right\}$ are identical mod $p$, hence the right-hand side of (10) is equal to $-\sum_{n=1}^{p-1} \cot \frac{n^{2} \pi}{p}$ and, by Whiteman's inequality (9), it is strictly negative. If $p \equiv 3(\bmod 8)$, the sets $\left\{-2 n^{2}\right\}$ and $\left\{n^{2}\right\}$ are identical $\bmod p$, therefore, the right-hand side of (10) is equal to $3 \sum_{n=1}^{p-1} \cot \frac{n^{2} \pi}{p}$, hence, by (9), it is strictly positive. Thus, in combination also with Property 1, we obtain the following:

Property 2. $T(p)>0$ if $p \equiv 3(\bmod 8)$ and $T(p)<0$ if $p \equiv 7(\bmod 8)$. Also, in the set $\{1,2, \ldots, p-1\}$, the odd quadratic residues $\bmod p$ are more than the even ones when $p \equiv 3(\bmod 8) ;$ the reverse situation is true when $p \equiv 7(\bmod 8)$.

Now consider the sum

$$
M(p)=-\sum_{k=1}^{p-1}\left(\frac{k}{p}\right) k .
$$

Dirichlet [10] proved that, for $p \equiv 3(\bmod 4), M(p)>0$, i.e. among the numbers $1,2, \ldots, p-1$, the sum of the quadratic non-residues is greater than the sum of the quadratic residues. In [3], B.C. Berndt proves that

$$
M(p)=\frac{\sqrt{p}}{2} \sum_{k=1}^{p-1}\left(\frac{k}{p}\right) \cot \frac{k \pi}{p}
$$

and, based on (11), he gives (Theorem 3.1 in [3]) another proof of Dirichlet's inequality

$$
M(p)>0 \quad \text { for } p \equiv 3 \quad(\bmod 4) .
$$

Using (11), it is an easy exercise to check that

$$
T(p)=\left\{\begin{array}{lll}
-M(p) & \text { if } p \equiv 7 & (\bmod 8) \\
3 M(p) & \text { if } p \equiv 3 & (\bmod 8)
\end{array}\right.
$$

This, combined with Property 2, gives now another proof of (12) which is simpler than that of Theorem 3.1 in [3].

An upper bound for $\boldsymbol{T}(\boldsymbol{p})$. From (7) we trivially obtain $|T(p)|<p(p-1) / 2$. However, we can obtain a much better upper bound as follows.

Let $Q \subseteq\{1,2, \ldots, p-1\}$ be a complete set of quadratic residues $\bmod p$. We have

$$
|T(p)| \leq \sqrt{p} \sum_{j \in Q}\left|\tan \frac{\pi j}{p}\right|=\sqrt{p} \sum_{j \in Q} \frac{1}{\left|\tan \frac{\pi(p-2 j)}{2 p}\right|} .
$$

Since $\left|\frac{\pi(p-2 j)}{2 p}\right|<\frac{\pi}{2}$, it follows that $\left|\tan \frac{\pi(p-2 j)}{2 p}\right|>\frac{\pi}{2 p}|p-2 j|$, hence,

$$
|T(p)|<\frac{2 p \sqrt{p}}{\pi} \sum_{j \in Q} \frac{1}{|p-2 j|} .
$$


Note that, as $j$ runs through the set $Q$, the numbers $|p-2 j|$ are distinct $\bmod p$, for, if $\left|p-2 j_{1}\right| \equiv\left|p-2 j_{2}\right|(\bmod p)$ with $j_{1}, j_{2} \in Q$ and $j_{1} \neq j_{2}$, then, necessarily, $j_{2} \equiv-j_{1}$ $(\bmod p)$, which implies that -1 is a quadratic residue $\bmod p$, a contradiction. Therefore, the set $\{|p-2 j|: j \in Q\}$ is a subset of $\{1, \ldots, p-1\}$ with cardinality $(p-1) / 2$, consisting of odd numbers, i. e. it coincides with $\{1,3, \ldots, p-2\}$. Therefore,

$$
\sum_{j \in Q} \frac{1}{|p-2 j|} \leq \sum_{k=1}^{(p-1) / 2} \frac{1}{2 k-1}<1+\frac{1}{2} \log (p-2),
$$

from which we obtain the following:

Property 3. For any prime $p \equiv 3(\bmod 4)$ we have

$$
|T(p)|<\frac{2 p \sqrt{p}}{\pi}\left(1+\frac{1}{2} \log (p-2)\right) .
$$

Now we go on to the study of $C(p)$. We use the following alternative expression for $C(p)$ (cf. (3)):

$$
C(p)=-i \sqrt{p} \sum_{j \in Q} \frac{1+\zeta^{j}}{1-\zeta^{j}}
$$

where $Q$ is a complete set of quadratic residues $\bmod p$. It is straightforward to check that $C(3)=1$, therefore we assume that $p>3$. By Whiteman's inequality (9), we have $C(p)>0$.

Just before obtaining Property 2, we actually proved that $T(p)=-C(p)$ if $p \equiv 7$ $(\bmod 8)$ and $T(p)=3 C(p)$ if $p \equiv 3(\bmod 8)$. Therefore, in view of $(13)$,

$$
C(p)=M(p) \text {. }
$$

Relations (13) and (16) combined with Property 1, imply the following:

Property 4. $C(p)$ is equal to the excess of the sum of quadratic non-residues over the sum of quadratic residues $\bmod p . C(p)$ is an odd positive integer, divisible by $p$ and by no higher power of $p$. If $p \equiv 3(\bmod 8)$, then $T(p)$ is a multiple of 3 , hence, $q_{o}(p)-q_{e}(p)$ is a positive multiple of 3 .

The following elegant property relates $T(p)$ with the class-number of the quadratic number field $\mathbb{Q}(\sqrt{-p})$.

Property 5. Let $p$ be a prime number $\equiv 3(\bmod 4)$ and let $h(-p)$ be the class-number of the quadratic number field $\mathbb{Q}(\sqrt{-p})$. Then,

$$
\begin{gathered}
T(p)= \begin{cases}-p h(-p) & \text { if } p \equiv 7 \quad(\bmod 8) \\
3 p h(-p) & \text { if } p \equiv 3 \quad(\bmod 8)\end{cases} \\
M(p)=C(p)=p h(-p) \\
h(-p)=\left\{\begin{array}{lll}
q_{e}(p)-q_{o}(p) & \text { if } p \equiv 7 \quad(\bmod 8) \\
\frac{1}{3}\left(q_{o}(p)-q_{e}(p)\right) & \text { if } p \equiv 3 & (\bmod 8)
\end{array}\right.
\end{gathered}
$$


Proof. We have

$$
h(-p)=\frac{1}{2 \sqrt{p}} \sum_{k=1}^{p-1}\left(\frac{k}{p}\right) \cot \frac{k \pi}{p} .
$$

This is a consequence of the more general formula, referred to as "Lebesgue's formula" in Dickson's "History" [9], p. 179, due to Dirichlet [11]. For a recent proof of that formula we refer the reader to the Corollary 2.3 of [5].

A complete set of quadratic non-residues $\bmod p$ is $\left\{-k^{2}: k=1, \ldots,(p-1) / 2\right\}$. Therefore, (20) becomes

$$
\begin{aligned}
h(-p) & =\frac{1}{2 \sqrt{p}}\left(\sum_{k=1}^{(p-1) / 2} \cot \frac{k^{2} \pi}{p}-\sum_{k=1}^{(p-1) / 2} \cot \frac{-k^{2} \pi}{p}\right) \\
& =\frac{1}{\sqrt{p}} \sum_{k=1}^{(p-1) / 2} \cot \frac{k^{2} \pi}{p}=\frac{1}{p} C(p)=\frac{1}{p} M(p)
\end{aligned}
$$

(by (16)), which proves (18), and now (17) and (19) are straightforward consequences of (13) and Property 1, respectively.

The relation (17) is a special case of Corollary 5.2 in [5] which goes back to V.A. Lebesgue [14]. The relation (18) is due to Dirichlet [11]; see also Corollary 3.6 of [5].

Note that, since $q_{e}(p)+q_{o}(p)=(p-1) / 2$, which is odd, Property 5 implies the following:

$$
\text { For a prime } p \equiv 3(\bmod 4), h(-p) \text { is odd. }
$$

This is Corollary 3.6 of [3].

Further expressions for $\boldsymbol{T}(\boldsymbol{p})$ and consequences. Since $\left(\frac{p-k}{p}\right)=-\left(\frac{k}{p}\right)$, we have from (6),

$$
T(p)=p \sum_{k=1}^{(p-1) / 2}(-1)^{k+1}\left(\frac{k}{p}\right) .
$$

We have

$$
\begin{aligned}
& \sum_{k=1}^{p-1}(-1)^{k+1}\left(\frac{k}{p}\right) \\
& \quad=\left(\frac{1}{p}\right)+\left(\frac{3}{p}\right)+\cdots+\left(\frac{p-2}{p}\right)-\left(\frac{2}{p}\right)-\left(\frac{4}{p}\right)-\cdots-\left(\frac{p-1}{p}\right) \\
& =\left(\frac{1}{p}\right)+\left(\frac{3}{p}\right)+\cdots+\left(\frac{p-2}{p}\right)+\left(\frac{p-2}{p}\right)+\left(\frac{p-4}{p}\right)+\cdots+\left(\frac{1}{p}\right) \\
& =2\left(\left(\frac{1}{p}\right)+\left(\frac{3}{p}\right)+\cdots+\left(\frac{p-2}{p}\right)\right)=2 A,
\end{aligned}
$$


where $A=\left(\frac{1}{p}\right)+\left(\frac{3}{p}\right)+\cdots+\left(\frac{p-2}{p}\right)$. Therefore, by (23) and (22),

$$
2 A=A-\left(\frac{2}{p}\right)\left(\left(\frac{1}{p}\right)+\left(\frac{2}{p}\right)+\cdots\left(\frac{(p-1) / 2}{p}\right)\right),
$$

implying

$$
A=-\left(\frac{2}{p}\right)\left(\left(\frac{1}{p}\right)+\left(\frac{2}{p}\right)+\cdots\left(\frac{(p-1) / 2}{p}\right)\right) .
$$

Collecting together the expressions for $T(p)$ in (6), (21), (23) and the expression for $A$ just above, we have:

$$
\begin{aligned}
T(p) & =\frac{p}{2} \sum_{k=1}^{p-1}(-1)^{k+1}\left(\frac{k}{p}\right) \\
& =p \sum_{k=1}^{(p-1) / 2}(-1)^{k+1}\left(\frac{k}{p}\right) \\
& =p\left(\left(\frac{1}{p}\right)+\left(\frac{3}{p}\right)+\cdots+\left(\frac{p-2}{p}\right)\right) \\
& =-p\left(\frac{2}{p}\right)\left(\left(\frac{1}{p}\right)+\left(\frac{2}{p}\right)+\cdots\left(\frac{(p-1) / 2}{p}\right)\right) .
\end{aligned}
$$

Note that the sum appearing in (27) is a sum of the values of a primitive character mod $p$, therefore, by the Pólya inequality ${ }^{1}$ (see Theorem 8.21 in [1] or inequality (2) in Chapter 23 of [8]) this sum is $<p^{1 / 2} \log p$. This gives the upper bound $|T(p)|<p^{3 / 2} \log p$, which is slightly worse than the upper bound in Property 3.

The expression (27) for $T(p)$, in combination with a well-known result of Dirichlet saying that, among the numbers $1,2, \ldots,(p-1) / 2$ there are more quadratic residues than nonquadratic residues mod $p$ (see e.g. [6], [19], [18], or exercises 14 through 17, Chapter 16 of [12]) furnishes another proof of Property 2.

Next, equating the right-hand sides of (25) and (27) and separating the Legendre symbols with even "numerators" from those with odd ones, we find

$$
\begin{aligned}
\left(1+\left(\frac{2}{p}\right)\right)\left(\left(\frac{1}{p}\right)+\left(\frac{3}{p}\right)+\cdots+\left(\frac{(p-1) / 2}{p}\right)\right) \\
=\left(1-\left(\frac{2}{p}\right)\right)\left(\left(\frac{2}{p}\right)+\left(\frac{4}{p}\right)+\cdots+\left(\frac{(p-3) / 2}{p}\right)\right) .
\end{aligned}
$$

If $p \equiv 3(\bmod 8)$, then (28) implies that $\left(\frac{2}{p}\right)+\left(\frac{4}{p}\right)+\cdots+\left(\frac{(p-3) / 2}{p}\right)=0$, hence, $\left(\frac{1}{p}\right)+\left(\frac{2}{p}\right)+\cdots+\left(\frac{(p-3) / 4}{p}\right)=0$, which says that, among the numbers $1,2, \ldots,(p-3) / 4$ there are as many quadratic residues as quadratic non-residues $\bmod p$; and since $T(p)>$

\footnotetext{
${ }^{1}$ Or Pólya-Vinogradov inequality.
} 
$0,(27)$ implies that there are more quadratic residues than quadratic non-residues among the numbers $(p+1) / 4, \ldots,(p-1) / 2$.

If $p \equiv 7(\bmod 8)$, then $(28)$ implies that $\left(\frac{1}{p}\right)+\left(\frac{3}{p}\right)+\cdots+\left(\frac{(p-1) / 2}{p}\right)=0$, hence, $\left(\frac{p-1}{p}\right)+\left(\frac{p-3}{p}\right)+\cdots+\left(\frac{(p+1) / 2}{p}\right)=0$ and, consequently,

$$
\left(\frac{(p+1) / 4}{p}\right)+\cdots+\left(\frac{(p-3) / 2}{p}\right)+\left(\frac{(p-1) / 2}{p}\right)=0 .
$$

This shows that there are as many quadratic residues as quadratic non-residues mod $p$ among the numbers $(p+1) / 4, \ldots,(p-1) / 2$; and since $T(p)<0,(27)$ implies that there are more quadratic residues than quadratic non-residues among the numbers $1, \ldots$, $(p-3) / 4$.

Property 6. If $p \equiv 3(\bmod 8)$ then, among the numbers $1,2, \ldots,(p-3) / 4$, there are as many quadratic residues as quadratic non-residues $\bmod p$ and among the numbers $(p+1) / 4, \ldots,(p-1) / 2$ the quadratic residues are more than the quadratic non-residues. If $p \equiv 7(\bmod 8)$ then, among the numbers $1,2, \ldots,(p-3) / 4$, there are more quadratic non-residues than quadratic residues $\bmod p$ and among the numbers $(p+1) / 4, \ldots$, $(p-1) / 2$ the quadratic residues are as many as the quadratic non-residues.

In other words,

$$
\begin{gathered}
\sum_{k=1}^{(p-3) / 4}\left(\frac{k}{p}\right)\left\{\begin{array}{lll}
>0 & \text { if } p \equiv 7 & (\bmod 8) \\
=0 & \text { if } p \equiv 3 & (\bmod 8)
\end{array}\right. \\
\sum_{k=(p+1) / 4}^{(p-1) / 2}\left(\frac{k}{p}\right) \quad\left\{\begin{array}{lll}
=0 & \text { if } p \equiv 7 & (\bmod 8) \\
>0 & \text { if } p \equiv 3 & (\bmod 8)
\end{array}\right.
\end{gathered}
$$

The relations (29) and (30) can also be inferred by an argument of B.C. Berndt and S. Chowla (p. 8 of [4]) in combination of their main Theorem therein, applied with $q=2$. Property 6 implies another interesting fact, already noted in 1979, namely,

Property 7. If $p \equiv 3(\bmod 8)$, then the number of even quadratic residues $\bmod p$ that are $<p / 2$ equals $(p-3) / 8$. If $p \equiv 7(\bmod 8)$, then the number of even quadratic residues that are $>p / 2$ equals $(p+1) / 8$.

The fact that Property 7 is implied by Property 6 is noted by Emma Lehmer [15].

Finally, we remark that our arguments that led to Property 6 furnish another expression for $T(p)$, namely,

$$
T(p)=\left\{\begin{array}{lll}
p\left(\left(\frac{(p+1) / 4}{p}\right)+\cdots+\left(\frac{(p-3) / 2}{p}\right)+\left(\frac{(p-1) / 2}{p}\right)\right) & \text { if } p \equiv 3 & (\bmod 8) \\
-p\left(\left(\frac{1}{p}\right)+\left(\frac{2}{p}\right) \cdots+\left(\frac{(p-3) / 4}{p}\right)\right) & \text { if } p \equiv 7 & (\bmod 8)
\end{array}\right.
$$




\section{References}

[1] T.M. Apostol, Introduction to Analytic Number Theory, Springer-Verlag, New York 1976.

[2] R.P. Bambah, S. Chowla, On the sign of the Gaussian sum, Proc. Nat. Inst. Sci. India 13 (1947), 175-176.

[3] B.C. BERndt, Classical theorems on quadratic residues, Enseign. Math. 22 (1976), 261-304.

[4] B.C. Berndt, S. Chowla, Zero sums of the Legendre symbol, Nordisk Mat. Tidskrift 22 (1974), 5-8.

[5] B.C. Berndt, A. Zaharescu, Finite trigonometric sums and class numbers, Math. Ann. 330 (2004), 551-575.

[6] Kai-Lai Chung, Note on a theorem on quadratic residues, Bull. Am. Math. Soc. 47 (1941), 514-516.

[7] H. Cohen, A Course in Computational Algebraic Number Theory, Springer Graduate Texts in Mathematics No 138, Berlin Heidelberg 1993.

[8] H. DavenPort, Multiplicative Number Theory - Second Edition, Graduate Texts in Mathematics Vol. 74, Springer-Verlag, New York 1980.

[9] L.E. Dickson, History of the Theory of Numbers, Vol. III, Chelsea Publishing Co., New York 1971.

[10] G.L. DiRichlet, Recherches sur diverses applications de l'analyse infinitésimale à la théorie des nombres, Reine Angew. Math. 19 (1839), 324-369.

[11] G.L. DiRICHLET, Recherches sur diverses applications de l' analyse infinitésimale à la théorie des nombres, seconde partie, J. Reine Angew. Math. 21 (1840), 134-155.

[12] K. Ireland, M. Rosen, A classical introduction to modern Number Theory, Graduate Texts in Mathematics Vol. 84, Springer-Verlag, New York 1982.

[13] HuA Loo Keng, Introduction to Number Theory, Springer Verlag, Berlin 1982.

[14] V.A. Lebesgue, Suite du Memoire sur les applications du symbole $\left(\frac{a}{b}\right)$, J. de Math. 15 (1850), $215-237$.

[15] E. Lehmer, Solution of Problem 6156, Am. Math. Monthly 86 No 2 (1979), 134-135.

[16] M. LERCH, Essais sur le calcul de nombre des classes de formes quadratiques binaires aux coefficients entiers, Acta Math. 29 (1905), 333-424; Acta Math. 30 (1906), 203-293.

[17] H.L. Montgomery, An exponential polynomial formed with the Legendre symbol, Acta Arith. 37 (1980), 375-380.

[18] L. Moser, A theorem on quadratic residues, Proc. Am. Math. Soc. 2 No 3 (1951), 503-504.

[19] A.L. Whiteman, Theorems on quadratic residues, Mathematics Magazine 23 No 2 (1949), 71-74.

A. Laradji

Department of Mathematics \& Statistics

KFUPM, Dhahran, Saudi Arabia

e-mail: alaradji@kfupm.edu.sa

M. Mignotte

Université Louis Pasteur, U. F. R. de Mathématiques

Strasbourg, France

e-mail: maurice@math.u-strasbg.fr

N. Tzanakis

Department of Mathematics, University of Crete

Iraklion, Greece

e-mail: tzanakis@math.uoc.gr

url: http: //www.math.uoc.gr/ utzanakis 